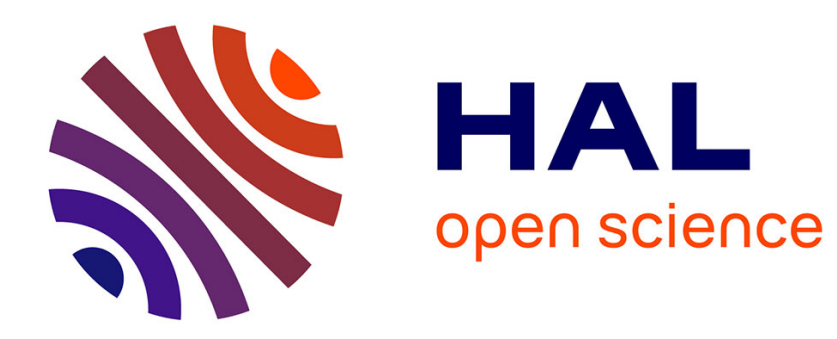

\title{
On smoothly growing bodies and the eshelby tensor
}

Reuven Segev

\section{To cite this version:}

Reuven Segev. On smoothly growing bodies and the eshelby tensor. Meccanica, 1996, 31 (5), pp.507518. hal-01064973

\section{HAL Id: hal-01064973 https://hal.science/hal-01064973}

Submitted on 17 Sep 2014

HAL is a multi-disciplinary open access archive for the deposit and dissemination of scientific research documents, whether they are published or not. The documents may come from teaching and research institutions in France or abroad, or from public or private research centers.
L'archive ouverte pluridisciplinaire HAL, est destinée au dépôt et à la diffusion de documents scientifiques de niveau recherche, publiés ou non, émanant des établissements d'enseignement et de recherche français ou étrangers, des laboratoires publics ou privés. 


\title{
On Smoothly Growing Bodies and the Eshelby Tensor
}

\author{
REUVEN SEGEV \\ Ben-Gurion University of the Negev, Department of Mechanical Engineering; \\ Beer-Sheva 84105, Israel
}

\begin{abstract}
A continuum mechanical theory of growing bodies is presented. It is assumed that the various parts of the body are identifiable. The growth of a body is manifested by mapping the identifiable elements of the growing body into a material manifold. Kinematics and stress theory are formulated on the basis of an infinite dimensional differentiable bundle structure for the configuration space. Stresses representing the forces associated with the growth of the body are analogous to the Eshelby tensor.
\end{abstract}

Sommario. Si propone una teoria meccanica dei corpi di massa crescente. Si Postula che le varie parti del copro siano identificabili. La crescita del corpo si manifesta mediante l'applicazione degli elementi identificabili del corpo in una varietà materiale. La cinematica e la teoria degli sforzi vengono formulati sulla base di un fibrato differenziabile a infinite dimensioni per lo spazio delle configurazioni. Gli sforzi associati alla crescita del corpo sono analoghi al tensore di Eshelby.

Key words: Growing bodies, Kinematics, Material velocity field, Force, Stress, Eshelby tensor.

\section{Introduction}

This work considers a theory of continuum mechanics in which the material structure of a body may vary. Material may be added to a body or removed from it. Our aim here is twofold. On the one hand we wish to present a mathematical model for the mechanical process in which a body grows. On the other hand, we wish to present a setting in which notions such as Eshelby's energy momentum tensor (Eshelby, 1969) and Gurtin's recent configurational forces (Gurtin, 1995) arise naturally. We note that neither Eshelby (Eshelby, 1969) nor Gurtin (Gurtin, 1995) has presented a systematic general continuum mechanics derivation of the corresponding forces and stresses.

In simple words, a growth of a body is reflected by a change of the undeformed, unloaded configuration of the body. In the sequel we will refer to such a state as the content of the body. We will make the notion of the content of a growing body more precise later. The situation of the body also changes by various deformations that it has in the physical space. Thus, in the kinematics of a growing body, both the reference configuration, and the configuration in space of the collection of material points are variable.

As is usually done in continuum mechanics, we will not assume specifically that bodies have undeformed unloaded configurations but will consider the contents of 
a growing body to be subsets of a material manifold. Each content of the body is a simple body of continuum mechanics and the configuration space of the growing body will be the union of the configuration spaces corresponding to the various contents of the body.

Additional structure is introduced because we want the have the possibility of telling "which part of the growing body has grown" and it might be helpful to use the growing human body as a metaphor. Thus, it is assumed that the various parts of the growing body are identifiable. In fact, our ability to identify the parts of the body will be extended all the way down to elements of a set - the growing body points.

The approach we use in formulating the kinematics, and force and stress theory is that of (Segev, 1984), (Segev, 1986) and (Segev and de Botton, 1991). Section 2 gives formal definitions for the notions discussed above and section 3 present the configuration space of the growing body with its differentiable structure as an infinite dimensional bundle. The base manifold of the bundle is the collection of contents of the growing body in the material manifold. The fiber at a particular content is the configuration space of the simple body corresponding to that content in space. Generalized velocities of the growing body are defined as elements of the tangent bundle to the configuration space. Section 4 considers the velocity fields of the material points that the growing body contains. Material velocity fields are elements of the vertical subbundle of the tangent bundle to the configuration space. As such, the mapping that provides the material velocity field associated with a generalized velocity of the growing body is a connection for the configuration space. Forces are defined as elements of the cotangent bundle and Section 5 discusses forces and their various decomposition into components associated with the vertical and horizontal subbundles. In Section 6 we consider forces that are continuous with respect to the $C^{1}$ norm. Such forces may be represented by stresses as in the results presented in (Segev, 1984) and (Segev and de Botton, 1991). The stresses representing the content-component of the force - the horizontal component - are those analogous to the Eshelby tensor.

\section{Growing Bodies and Their Contents}

In this section we give a mathematical framework that describes the kinematics of growing bodies. By growing bodies we roughly mean phenomena in which the region occupied by a body in space changes not only because of deformation but also because of the addition of material points to the body. When considering the addition of material points we have in mind a process like the growth of a plant leaf or the growth of the human body through a process of division of cells. Of course, we want to suggest a continuum model so one may think of the cells as being infinitesimally small. Alternatively, if one is willing to assume that bodies have an unloaded reference configuration is space, it is possible to think of the growth 
of the body as an evolution of the unloaded reference state. In order to have a clear distinction between growing bodies and the regular bodies in continuum mechanics - those containing a fixed collection of material points - we will refer to the latter as simple bodies. The mathematical model of the notion of a growing body has to convey the idea that, like the case of the growing human body, we can assign names to the body and its parts, the subbodies, even though they do not contain a "fixed number of material points". Thus it is assumed that we have a growing body $\mathcal{B}$ and a collection of growing subbodies and a partial ordering on this collection which lets us specify that one particular subbody $\mathcal{P}_{1}$ is contained in another growing subbody $\mathcal{P}_{2}$. Here, $\mathcal{B}, \mathcal{P}_{1}$, and $\mathcal{P}_{2}$, etc. are merely elements in the collection of the growing subbodies and do not have any additional mathematical structure. Next, we wish to assume that our ability to identify growing subbodies does not stop at a certain scale. Rather, it is possible to construct sequences of growing subbodies contained in one another that decrease in size whose limits can be identified with elements of a set. This provides $\mathcal{B}$ with the structure of a set and we will refer to its elements as the growing body points. The various growing subbodies are subsets of $\mathcal{B}$. In other words, we assume that the body can be divided into smaller and smaller identifiable portions whose limits, the undividable portions or "atoms" are the growing body points. A continuous variation of the properties of the growing body points may serve as a motivation for assuming that they are identifiable. These assumptions can be put into a more rigorous framework using Boolean algebras and Stone's representation theorem (Dunford and Schwartz, 1971, p. 43, for example). Further, we add geometrical structure by assuming that the growing body $\mathcal{B}$ and its subbodies have the structure of three dimensional, compact differentiable manifolds with boundaries of a three dimensional Euclidean space.

As stated, our objective is to model the growth of a growing body so material may be added or removed from it. We do this by assuming that there is a large collection of material points, $M$, to which we will refer as the material manifold and whose elements will be referred to as material points. We assume that $M$ is a three dimensional Euclidean space with tangent space V. At any stage in the growth of the growing body, the material points it contains are the image of a mapping $c: \mathcal{B} \rightarrow M$. Since the growing subbodies and body elements are identifiable in any of the stages of growth, we assume that $c$ is an embedding. We will refer to such an embedding $\mathcal{B} \rightarrow M$ as a content of $M$. Thus, the image of a content $c$ is a simple body.

For a given growing body $\mathcal{B}$, we will use $\operatorname{Emb}(\mathcal{B}, M)$ to denote the collection of embeddings of $\mathcal{B}$ in $M$. Hence, the collection of all contents of $\mathcal{B}$, the content space, can be identified with $\operatorname{Emb}(\mathcal{B}, M)$. We recall (Michor, 1980) that the collection of $C^{\omega}$ embeddings $\operatorname{Emb}(\mathcal{B}, M)$ is an open subset of $C^{\omega}(\mathcal{B}, M)$, the collection of all $C^{\omega}$ mappings for $\omega=1,2, \ldots, \infty$. Hence, for $\omega \geq 1$, the content space is a (trivial) differentiable manifold. The tangent space $T_{c} \operatorname{Emb}(\mathcal{B}, M)$ to the content 
space at any content $c$ can be identified with $C^{\omega}(\mathcal{B}, \mathbf{V})$. We will refer to an element $\dot{c} \in T \operatorname{Emb}(\mathcal{B}, M)$ as a growth rate.

\section{The Configuration Space of a Growing Body}

For a content $c$ in $\operatorname{Emb}(\mathcal{B}, M), B=c\{\mathcal{B}\}$ is a simple body. Thus, one may consider its configurations in space. We recall that a configuration $\kappa$ of a simple body $B$ is an embedding $\kappa: B \rightarrow \Re^{3}$, where for the sake of simplicity we model the physical space by $\Re^{3}$. We will denote by $Q_{c\{\mathcal{B}\}}$ the configuration space of the simple body $c\{\mathcal{B}\}$ in space, hence, $Q_{c\{\mathcal{B}\}}=\operatorname{Emb}\left(c\{\mathcal{B}\}, \Re^{3}\right)$. It will be useful sometimes to regard a configuration $\kappa \in Q_{c\{\mathcal{B}\}}$ as a mapping

$$
\hat{\kappa}: c(\mathcal{B}) \subset M \rightarrow M \times \Re^{3}, \quad \hat{\kappa}=(1, \kappa) .
$$

DEFINITION 1. The configuration space of the growing body $\mathcal{B}$ is

$$
Q_{\mathcal{B}}=\bigcup_{c} Q_{c\{\mathcal{B}\}}, \quad c \in \operatorname{Emb}(\mathcal{B}, M)
$$

The mapping

$$
\pi: Q_{\mathcal{B}} \rightarrow \operatorname{Emb}(\mathcal{B}, M)
$$

defined by $\pi(\kappa)=c$ if $\kappa \in Q_{c\{\mathcal{B}\}}$ will be referred to as the configuration space projection.

Consider a typical content $c_{0}$ of $\mathcal{B}$. The fiber $\pi^{-1}\left\{c_{0}\right\}=Q_{c_{0}\{\mathcal{B}\}}$, being a space of embeddings, is an open subset of $C^{\omega}\left(c_{0}\{\mathcal{B}\}, \Re^{3}\right), \omega \geq 1$. Hence, it is a differentiable manifold whose tangent space at any configuration $\kappa \in Q_{c_{0}\{\mathcal{B}\}}$ can be identified with $C^{\omega}\left(c_{0}\{\mathcal{B}\}, \Re^{3}\right)$.

In order to construct the kinematics of growing bodies, one has to endow $Q_{\mathcal{B}}$ with the structure of a differentiable manifold.

PROPOSITION 2. The configuration space of the growing body $Q_{\mathcal{B}}$ has the structure of a trivializable differentiable bundle.

Proof. Let $c_{0}$ be a content of the body. Then, since for any other content $c$, $c\{\mathcal{B}\}$ is diffeomorphic to $c_{0}\{\mathcal{B}\}$, the manifolds $Q_{c_{0}\{\mathcal{B}\}}$ and $Q_{c\{\mathcal{B}\}}$ are diffeomorphic. In fact, the diffeomorphism may be explicitly written as $\kappa \mapsto \kappa_{0}=\kappa \circ c \circ c_{0}^{-1}, \kappa \in$ $Q_{c\{\mathcal{B}\}}, \kappa_{0} \in Q_{c_{0}\{\mathcal{B}\}}$. We will refer to such a trivialization of $Q_{\mathcal{B}}$ as a trivialization centered at $c_{0}$. For a given content $c_{1}$, the representative $\kappa_{1}$ of a configuration $\kappa \in Q_{c\{\mathcal{B}\}}$ under the chart centered at $c_{1}$ is related to $\kappa_{0}$ by $\kappa_{1}=\kappa_{0} \circ c_{0} \circ c_{1}^{-1}$. This last relation is clearly differentiable.

It follows that for arbitrary contents $c_{0}, c_{1}$, we have

$$
\kappa_{1} \circ c_{1}=\kappa_{0} \circ c_{0}=\kappa \circ c .
$$


In the sequel, $e=\kappa \circ c$ will be referred to a the extent of the growing body at the configuration $\kappa$. Clearly, the extent of the body is an embedding of $\mathcal{B}$ in $\Re^{3}$ that is independent of the choice of a chart. It is obvious that the mapping $\Phi: Q_{\mathcal{B}} \rightarrow$ $\operatorname{Emb}(\mathcal{B}, M) \times \operatorname{Emb}\left(\mathcal{B}, \Re^{3}\right)$ given by $\Phi(\kappa)=(\pi(\kappa), \kappa \circ c)=(c, e)$ is a bijection. Thus, $\Phi$ generates a homeomorphism of $Q_{\mathcal{B}}$ with $\operatorname{Emb}(\mathcal{B}, M) \times \operatorname{Emb}\left(\mathcal{B}, \Re^{3}\right)$ so that a configuration $\kappa$ may be represented by a mapping $(c, e): \mathcal{B} \rightarrow M \times \Re^{3}$.

A motion of the growing body is a differentiable mapping $\Re \rightarrow Q_{\mathcal{B}}$ and a generalized velocity at the configuration $\kappa \in Q_{\mathcal{B}}$ is an element of $T_{\kappa} Q_{\mathcal{B}}$, i.e., it is a tangent to a motion passing through $\kappa$.

Under a chart centered at the content $c_{0}$ elements of $Q_{\mathcal{B}}$ are represented by ordered pairs of the form $\left(c, \kappa_{0}\right) \in \operatorname{Emb}(\mathcal{B}, M) \times Q_{c_{0}\{\mathcal{B}\}}$. Hence, under this chart generalized velocities will be represented in the form $\left(c, \kappa_{0}, \dot{c}, \dot{\kappa}_{0}\right) \in \operatorname{Emb}(\mathcal{B}, M) \times$ $Q_{c_{0}\{\mathcal{B}\}} \times T_{c} \operatorname{Emb}(\mathcal{B}, M) \times T_{\kappa_{0}} Q_{c_{0}\{\mathcal{B}\}}$.

PROPOSITION 3. If $\left(c, \kappa_{0}, \dot{c}, \dot{\kappa}_{0}\right)$ in

$$
\operatorname{Emb}(\mathcal{B}, M) \times Q_{c_{0}\{\mathcal{B}\}} \times T_{c} \operatorname{Emb}(\mathcal{B}, M) \times T_{\kappa_{0}} Q_{c_{0}\{\mathcal{B}\}}
$$

represents a generalized velocity $\dot{\kappa}$ using a chart centered at $c_{0}$ and $\left(c, \kappa_{1}, \dot{c}, \dot{\kappa}_{1}\right)$ represents $\dot{\kappa}$ using a chart centered at $c_{1}$, then the transformation rule is $\dot{\kappa}_{0} \circ c_{0}=$ $\dot{\kappa}_{1} \circ c_{1}$.

Proof. The transformation rule for the derivatives is obtain by differentiating the relation $\kappa_{1}(t) \circ c_{1}=\kappa_{0}(t) \circ c_{0}$ with respect to the "time" parameter.

The collection of all generalized velocities of the growing body is therefore the tangent bundle $T Q_{\mathcal{B}}$ on which one has the tangent bundle projection $\tau_{Q_{\mathcal{B}}}: T Q_{\mathcal{B}} \rightarrow$ $Q_{\mathcal{B}}$ assigning to any generalized velocity $\dot{\kappa}$ the configuration at which it is tangent. Another natural mapping defined on the tangent bundle $T Q_{\mathcal{B}}$ is the tangent to the configuration space projection $T \pi: T Q_{\mathcal{B}} \rightarrow T \operatorname{Emb}(\mathcal{B}, M)$ that assigns to each generalized velocity of the growing body the corresponding growth rate. Locally, $\tau_{Q_{\mathcal{B}}}$ is represented by $\left(c, \kappa_{0}, \dot{c}, \dot{\kappa}_{0}\right) \mapsto\left(c, \kappa_{0}\right)$ while $T \pi$ is represented locally by $\left(c, \kappa_{0}, \dot{c}, \dot{\kappa}_{0}\right) \mapsto(c, \dot{c})$.

PROPOSITION 4. The mapping $\Phi$ is differentiable. The representative of the tangent $T \Phi$ maps $\dot{\kappa}$ into $(\dot{c}, \dot{e})=\left(\dot{c}, \dot{\kappa}_{0} \circ c_{0}\right)$ for a chart centered at the content $c_{0}$.

Proof. We use a chart centered at some content $c_{0}$. A generalized motion of the growing body is represented by curves $c(t), \kappa_{0}(t)$ in $\operatorname{Emb}(\mathcal{B}, M)$ and $Q_{c_{0}\{\mathcal{B}\}}$, respectively. Since $\kappa(t)=\kappa_{0}(t) \circ c_{0} \circ c(t)^{-1}$, we have

$$
\begin{aligned}
\Phi(\kappa(t)) & =(c(t), \kappa(t) \circ c(t)) \\
& =\left(c(t), \kappa_{0}(t) \circ c_{0} \circ c(t)^{-1} \circ c(t)\right) \\
& =\left(c(t), \kappa_{0}(t) \circ c_{0}\right) .
\end{aligned}
$$


The first component is clearly differentiable and the time derivative $\dot{e}$ of the extent is therefore given by $\dot{e}=\dot{\kappa}_{0} \circ c_{0}$. Hence, $\Phi$ is differentiable and in fact, it generates a global differentiable bundle chart on $Q_{\mathcal{B}}$.

The fact that $\Phi$ generates a natural global chart on $Q_{\mathcal{B}}$ valued in $\operatorname{Emb}(\mathcal{B}, M) \times$ $\operatorname{Emb}\left(\mathcal{B}, \Re^{3}\right)$ suggests a geometrical interpretation of the space of generalized velocities $T_{\kappa} Q_{\mathcal{B}}$ as follows. The tangent $T_{\kappa} \Phi$ to $\Phi$ at the configuration $\kappa$ of the growing body, generates a natural isomorphism of $T_{\kappa} Q_{\mathcal{B}}$ with $T_{c} \operatorname{Emb}(\mathcal{B}, M) \times T_{e} \operatorname{Emb}\left(\mathcal{B}, \Re^{3}\right)$ with $e=\kappa \circ c$. Now, the tangent space $T_{c} \operatorname{Emb}(\mathcal{B}, M)$ may be identified with $C^{\omega}(\mathcal{B}, \mathbf{V})$ and $T_{e} \operatorname{Emb}\left(\mathcal{B}, \Re^{3}\right)$ may be identified with $C^{\omega}\left(\mathcal{B}, \Re^{3}\right)$, hence, $T_{\kappa} Q_{\mathcal{B}}$ is naturally isomorphic to $C^{\omega}\left(\mathcal{B}, \mathbf{V} \times \Re^{3}\right)$. In other words, for each generalized velocity of the growing body there is a unique vector field defined on $\mathcal{B}$ and valued in $\mathbf{V} \times \Re^{3}$. The element of $C^{\omega}\left(\mathcal{B}, \mathbf{V} \times \Re^{3}\right)$ representing $\dot{\kappa}$ may be thought of as a variation $(\dot{c}, \dot{e}): \mathcal{B} \rightarrow T\left(M \times \Re^{3}\right)$ of the mapping $(c, e): \mathcal{B} \rightarrow M \times \Re^{3}$.

\section{Material Velocity Fields}

The representation of generalized velocities of the growing body and the transformation rule for their representatives presented in Proposition 3, make it clear that while the element $\dot{c} \in T_{c} \operatorname{Emb}(\mathcal{B}, M)$ is uniquely determined by the generalized velocity $\dot{\kappa}$, the other component of the representative, $\dot{\kappa}_{0} \in T_{\kappa_{0}} Q_{c_{0}\{\mathcal{B}\}}$, depends on the choice of content $c_{0}$. We recall that $\dot{\kappa}_{0}$ describes a velocity of the simple body $c_{0}\{\mathcal{B}\}$ and as such it has a clearer kinematical meaning than $\dot{\kappa}$. In the sequel we will refer to the velocity of a simple body as a material velocity (field). We conclude therefore that the representatives $\left(\dot{c}, \dot{\kappa}_{0}\right)$ do not provide an invariant decomposition of the generalized velocity $\dot{\kappa}$ into a growth rate and a material velocity field. While $(\dot{c}, \dot{e}) \in T_{c} \operatorname{Emb}(\mathcal{B}, M) \times T_{e} \operatorname{Emb}\left(\mathcal{B}, \Re^{3}\right)$ is uniquely defined by $\dot{\kappa}$, the component $\dot{e}$ does not have the usual meaning of a material velocity field. In this section we discuss the various aspects of an invariant decomposition of generalized velocities into growth rates and material velocity fields. Mathematically, such a decomposition is a connection on the bundle $Q_{\mathcal{B}}$.

Consider $\kappa \in Q_{c\{\mathcal{B}\}}$. An element $\dot{\kappa}$ of $T_{\kappa} Q_{\mathcal{B}}$ will be termed vertical if $T \pi(\dot{\kappa})=0$ so that no growth of the body is associated with it. Such a vertical generalized velocity can be regarded as an element of $T_{\kappa} Q_{c\{\mathcal{B}\}}$ and thus it represents a material velocity field.

To motivate the geometrical construction of the connection, consider a motion $(c(t), e(t))$ in $\operatorname{Emb}(\mathcal{B}, M) \times \operatorname{Emb}\left(\mathcal{B}, \Re^{3}\right)$ whose tangent at $t=0$ is $(\dot{c}, \dot{e})$. If the material point $X$ is in the interior of $B=c(\mathcal{B})$, then, there is a neighborhood $U$ of the zero in $\Re$ for which $c(t)^{-1}(X)$ is well defined so that $e(t) \circ c(t)^{-1}(X)$ is a motion of the material point $X$ in space. Let $\mathbf{v}(X)$ be the tangent to this motion so that

$$
\mathbf{v}(X)=\dot{e} \circ c^{-1}(X)+\left(\mathrm{D} e \circ c^{-1}\right)\left(\frac{d}{d t}\left((c(t))^{-1}\right)(X)\right),
$$


where, De denotes the derivative (gradient) of $e$. Using

$$
\frac{d}{d t}\left((c(t))^{-1}(X)\right)=-\left(\mathrm{D} c^{-1}(X)\right)\left(\dot{c} \circ c^{-1}(X)\right)
$$

we arrive at

$$
\mathbf{v}(X)=\dot{e} \circ c^{-1}(X)-\left(\operatorname{D} e \circ c^{-1}(X)\right)\left(\mathrm{D}^{-1} \circ c^{-1}(X)\right)\left(\dot{c} \circ c^{-1}(X)\right)
$$

so that

$$
\mathbf{v}(X)=\dot{e} \circ c^{-1}(X)-\mathrm{D} \kappa(X)\left(\dot{c} \circ c^{-1}(X)\right) .
$$

This relation suggests that the material velocity may be defined for the interior of the body. Two problems are apparent in this expression: The material velocity field is defined only for the interior of the body and the appearance of $\mathrm{D} \kappa$ in the expression implies that the material velocity field is $C^{k-1}$ if $\kappa$ is $C^{k}$.

To construct the connection geometrically, it is useful to use the mapping $\hat{\kappa}$ when considering the decomposition of $T_{\kappa} Q_{\mathcal{B}}$. We will also restrict ourselves to the case where $\kappa$ is $C^{\infty}$. Using the natural global chart $\Phi$, let

$$
\hat{\Gamma}: T_{c} \operatorname{Emb}(\mathcal{B}, M)=C^{\infty}(\mathcal{B}, \mathbf{V}) \rightarrow C^{\infty}\left(\mathcal{B}, \mathbf{V} \times \Re^{3}\right)=T_{\kappa} \Phi\left\{T_{\kappa} Q_{\mathcal{B}}\right\}
$$

be given by $\hat{\Gamma}(\dot{c})=(1, \mathrm{D} \kappa \circ c)(\dot{c})$, i.e., $\hat{\Gamma}(\dot{c})(\xi)=\left(\dot{c}(\xi)\right.$, D $\left.\kappa_{c(\xi)}(\dot{c}(\xi))\right)$. Thus, $\hat{\Gamma}$ maps $\dot{c}$ into a vector field tangent to the image of $\hat{\kappa}$ (the graph of $\kappa$ ). The mapping

$$
T_{\kappa} \Phi^{-1} \circ \hat{\Gamma}: T_{\pi(\kappa)} \operatorname{Emb}(\mathcal{B}, M) \rightarrow T_{\kappa} Q_{\mathcal{B}}
$$

will be denoted by $\Gamma$. From its local expression it is clear that $T \pi \circ \Gamma=1$.

Next, note that

$$
1-\hat{\Gamma} \circ T \pi: T_{c} \operatorname{Emb}(\mathcal{B}, M) \times T_{e} \operatorname{Emb}\left(\mathcal{B}, \Re^{3}\right) \rightarrow T_{c} \operatorname{Emb}(\mathcal{B}, M) \times T_{e} \operatorname{Emb}\left(\mathcal{B}, \Re^{3}\right)
$$

satisfies

$$
(1-\hat{\Gamma} \circ T \Phi)(\dot{c}, \dot{e})=(0, \dot{e}-(\mathrm{D} \kappa \circ c)(\dot{c}))
$$

Hence, we define

$$
\hat{\Delta}: T_{c} \operatorname{Emb}(\mathcal{B}, M) \times T_{e} \operatorname{Emb}\left(\mathcal{B}, \Re^{3}\right) \rightarrow T_{e} \operatorname{Emb}\left(\mathcal{B}, \Re^{3}\right)
$$

by $\hat{\Delta}=\operatorname{pr}_{2} \circ(1-\hat{\Gamma} \circ T \pi)=\dot{e}-(\mathrm{D} \kappa \circ c)(\dot{c})$. By its definition, $\hat{\Delta}(\dot{c}, \dot{e})$ represents a material velocity field. The expression for $\hat{\Delta}$ is analogous to the expression obtained above for $\mathbf{v}$ only the former is defined on $\mathcal{B}$ and latter is defined on $c\{\mathcal{B}\}$. Hence, letting $c^{-1 *}: T_{e} \operatorname{Emb}\left(\mathcal{B}, \Re^{3}\right) \rightarrow T_{\kappa} Q_{c\{\mathcal{B}\}}$ be given by $c^{-1 *}(\dot{e})=\dot{e} \circ c^{-1}$, we define

$$
\Delta: T_{\kappa} Q_{\mathcal{B}} \rightarrow T_{\kappa} Q_{c\{\mathcal{B}\}}
$$

by

$$
\Delta=c^{-1 *} \circ \hat{\Delta} \circ T \Phi .
$$


Thus, $\Delta$ assigns to any generalized velocity a material velocity field whose local expression is identical to the one presented previously. Clearly, if $\dot{\kappa}$ is in $T_{\kappa} Q_{c\{\mathcal{B}\}} \subset$ $T_{\kappa} Q_{\mathcal{B}}$, then $\Delta(\dot{\kappa})=\dot{\kappa}$. In addition we have, $T \pi \circ$ Inclusion $\circ \Delta=0, \Delta \circ \Gamma=0$, where Inclusion: $T_{\kappa} Q_{c\{\mathcal{B}\}} \rightarrow T_{\kappa} Q_{\mathcal{B}}$ is the inclusion mapping. We conclude that $\Delta$ and $T \pi$ decompose the collection of velocity fields into material velocity fields and growth rates. The situation is illustrated by the following diagram.

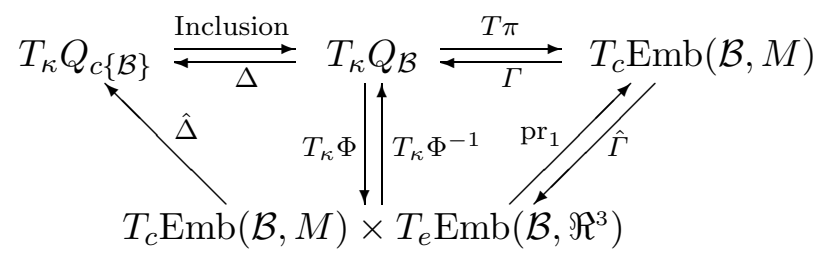

\section{Forces and Their Decompositions}

In this section we present the basic properties of forces as implied by the structure of the configuration space for the growing body and the definition of forces as elements of the cotangent bundle $T^{*} Q_{\mathcal{B}}$ of the configuration space. Thus, a force at the configuration $\kappa$ of the growing body is a continuous linear mapping $f_{\mathcal{B}}: T_{\kappa} Q_{\mathcal{B}} \rightarrow$ $\Re$. We will use simple body force for forces on bodies with a fixed material structure. In addition, we have forces corresponding to the content space of the body and we will refer to a functional $f_{c}: T_{c} \operatorname{Emb}(\mathcal{B}, M) \rightarrow \Re$ as a content force.

We note that a content force $f_{c}$ induces a force $f_{\mathcal{B}}$ on the growing body by $f_{\mathcal{B}}=T \pi^{*}\left(f_{c}\right)$. Here, $T \pi^{*}$ is the dual of the mapping $T \pi$ so $f_{\mathcal{B}}(\dot{\kappa})=f_{c}(T \pi(\dot{\kappa}))$. Similarly, a force on a growing body $f_{\mathcal{B}}$ at the content $c$ of $\mathcal{B}$ induces a force $f_{m}$ on the simple body $c\{\mathcal{B}\}$ by $f_{m}(\mathbf{v})=f_{\mathcal{B}}(\operatorname{Inclusion}(\mathbf{v}))$.

The global chart $\Phi$ introduced in Section 3, can be used in order to represent forces on growing bodies by two components: a content force and and extent forcean element of the dual space $T_{e}^{*} \operatorname{Emb}\left(\mathcal{B}, \Re^{3}\right)$. Thus,

$$
T_{\kappa}^{*} \Phi: T_{c}^{*} \operatorname{Emb}(\mathcal{B}, M) \times T_{e}^{*} \operatorname{Emb}\left(\mathcal{B}, \Re^{3}\right) \rightarrow T_{\kappa}^{*} Q_{\mathcal{B}}, \quad e=\kappa \circ c
$$

satisfies $f_{\mathcal{B}}(\dot{\kappa})=T_{\kappa}^{*} \Phi\left(f_{c}, f_{e}\right)(\dot{\kappa})=f_{c}(\dot{c})+f_{e}(\dot{e})$ where $(\dot{c}, \dot{e})$ are the representatives of the generalized velocity $\dot{\kappa}$ under the chart $\Phi$. Since $T_{\kappa} \Phi$ is an isomorphism, the last relation also gives the content force and extent force that represent a given force $f_{\mathcal{B}}$ on the growing body.

The content force represents the power expanded when the extent rate vanishes momentarily. The power corresponding to the rate of change of the "unloaded configuration" is evaluated. Thus, it includes power pertaining to the motion of the material points that get crowded as material is added into a fixed region in space. The extent force represents the power expanded when the growth rate vanishes momentarily, however, the velocity for which the power is computed is the extent 
rate and not the material velocity when $\dot{c} \neq 0$. Thus, the extent force performs power even in cases where the material velocity field vanishes, i.e., $\dot{e}=\mathrm{D} \kappa(\dot{c})$.

An additional representation of forces on growing bodies is provided by the decomposition of generalized velocities into growth rates and material velocities described in Section 4. Recalling that

$$
(T \pi, \Delta): T_{\kappa} Q_{\mathcal{B}} \rightarrow T_{\pi(\kappa)} \operatorname{Emb}(\mathcal{B}, M) \times T_{\kappa} Q_{c\{\mathcal{B}\}}
$$

is an isomorphism whose inverse is $\Gamma \circ \mathrm{pr}_{1}+$ Inclusion $\circ \mathrm{pr}_{2}$. It follows that

$$
(T \pi, \Delta)^{*}:\left(T_{\pi(\kappa)} \operatorname{Emb}(\mathcal{B}, M) \times T_{\kappa} Q_{c\{\mathcal{B}\}}\right)^{*} \rightarrow T_{\kappa}^{*} Q_{\mathcal{B}}
$$

is also an isomorphism. Hence, a force $f_{\mathcal{B}}$ may be represented by a force $f_{a} \in$ $T_{c}^{*} \operatorname{Emb}(\mathcal{B}, M)$ and a simple body force $f_{m}$ in the form

$$
f_{\mathcal{B}}(\dot{\kappa})=\left[(T \pi, \Delta)^{*}\left(f_{a}, f_{e}\right)\right](\dot{\kappa})=f_{a}(\dot{c})+f_{m}(\mathbf{v}), \quad \dot{c}=T \pi(\dot{\kappa}), \mathbf{v}=\Delta(\dot{\kappa}) .
$$

Note that the force $f_{a}$ represents the power performed by the force on the growing body in the case where the material velocity vanishes. Thus, it represents the power expanded by the force in the case of pure growth, i.e., when $\dot{e}=\mathrm{D} \kappa(\dot{c})$.

One can relate the representation of a force on a growing body using the global chart $\Phi$ and the representation using the connection. We have

$$
\begin{aligned}
f_{\mathcal{B}}(\dot{\kappa})=f_{c}(\dot{c})+f_{e}(\dot{e}) & =f_{a}(\dot{c})+f_{m}(\mathbf{v}) \\
& =f_{a}(\dot{c})+f_{m}\left(\dot{e} \circ c^{-1}-\left(\mathrm{D} \kappa \circ c^{-1}\right)\left(\dot{c} \circ c^{-1}\right)\right) \\
& =\left(f_{a}-f_{m} \circ\left(\mathrm{D} \kappa \circ c^{-1}\right) \circ c^{-1 *}\right)(\dot{c})+f_{m} \circ c^{-1 *}(\dot{e}),
\end{aligned}
$$

where, $c^{-1 *}$ is the pullback of vector fields defined on $\mathcal{B}$ into vector fields defined on $c\{\mathcal{B}\}$ so $c^{-1 *}(\dot{c})=\dot{c} \circ c^{-1}, c^{-1 *}(\dot{e})=\dot{e} \circ c^{-1}$ and we used the expression for the material velocity field. Thus, the relations obtained is

$$
\begin{aligned}
& f_{c}=f_{a}-f_{m} \circ\left(\mathrm{D} \kappa \circ c^{-1}\right) \circ c^{-1 *} \\
& f_{e}=f_{m} \circ c^{-1 *} .
\end{aligned}
$$

The inverse relations may be obtained using $\dot{e}=\mathbf{v} \circ c+(\mathrm{D} \kappa \circ c)(\dot{c})$ as

$$
\begin{aligned}
f_{a} & =f_{c}+f_{e} \circ(\mathrm{D} \kappa \circ c) \\
f_{m} & =f_{e} \circ c^{*} .
\end{aligned}
$$

The situation is illustrated in the following diagram.

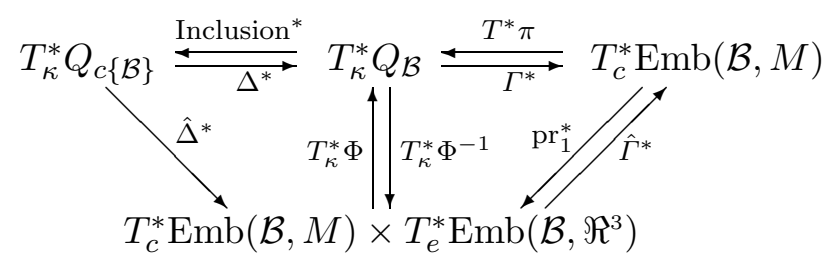


A mixed representation of the force $f_{\mathcal{B}}$ can be obtained by substituting the expression for $f_{a}$ into the representation $f_{\mathcal{B}}(\dot{\kappa})=f_{a}(\dot{c})+f_{e}(\dot{e})$ to obtain $f_{\mathcal{B}}(\dot{\kappa})=$ $\left(f_{c}+f_{e} \circ(\mathrm{D} \kappa \circ c)\right)(\dot{c})+f_{m}(\mathbf{v})$. Using the equation for $f_{e}$ in terms of $f_{m}$, we finally arrive at

$$
f_{\mathcal{B}}(\dot{\kappa})=\left(f_{c}+f_{m} \circ c^{-1 *} \circ(\mathrm{D} \kappa \circ c)\right)(\dot{c})+f_{m}(\mathbf{v}) .
$$

This representation uses the physically significant $f_{m}$ and content force $f_{c}$ describing the power expanded as the "reference unloaded configuration" changes.

\section{Stress Representation of Forces}

This section considers basic stress theory for growing bodies. In accordance with (Segev, 1984),(Segev, 1986) and (Segev and de Botton, 1991), the basic framework in which stresses are defined is the representation of continuous linear functionals on $C^{\omega}(D, \mathbf{W})$ for a finite $\omega$, where $D$ is a compact three dimensional submanifold with boundary of a three dimensional Euclidean space $E$ with tangent space is $T_{E}$, and $\mathbf{W}$ is a finite dimensional vector space. We note that as $D$ is compact, any element $f$ of $C^{\infty}(D, \mathbf{W})$ is of a finite order, i.e., $f \in C^{k}(D, \mathbf{W})$ for a finite $k$. In particular, stress theory for continuum mechanics of order one is obtained if we use $\omega=1$ as summarized by the following proposition.

PROPOSITION 5. A continuous linear functional $f \in C^{1}(D, \mathbf{W})^{*}$ can be represented by measures $\sigma$ and $\Sigma$ defined on $D$ and valued in $\mathbf{W}$ and $L\left(\mathbf{W}, T_{E}\right)$, respectively, in the form

$$
f(u)=\int_{D} u \cdot d \sigma+\int_{D} \mathrm{D} u \cdot d \Sigma, \quad u \in C^{1}(D, \mathbf{W}) .
$$

The measure $\sigma$ is referred to as the ambient force or the self force and in case one assumes that the total force on each subbody of $D$ vanishes then $\sigma=0$. The tensor measure $\Sigma$ is referred to as the stress tensor. The representation of a force by stresses is not unique, i.e., there is more then one pair $(\sigma, \Sigma)$ that represents a generalized force $f$.

If a force $f_{P}$ is given for any subbody $P$ of $D$ such that

$$
f_{P}(u)=\int_{P} u \cdot d \sigma+\int_{P} \mathrm{D} u \cdot d \Sigma, \quad u \in C^{1}(P, \mathbf{W}),
$$

for some stress measures $\sigma$ and $\Sigma$, then the stress measures are unique.

In the case where the stress measures $\sigma$ and $\Sigma$ can be represented by differentiable densities $s$ and $S$ with respect to the volume measure $V$ on $D$ so the representation of the force $f$ is in the form

$$
f(u)=\int_{D} u \cdot s d V+\int_{D} \mathrm{D} u \cdot S d V, \quad u \in C^{1}(D, \mathbf{W}),
$$


there are unique vector fields $b$ defined on $D$ and $t$ defined on $\partial D$-the usual body force and surface force-related to the stress densities by $\operatorname{Div} S+b=s$ on $D$ and $S(n)=\sigma$ on $\partial D$, such that

$$
f(u)=\int_{D} u \cdot b d V+\int_{\partial D} u \cdot t d A, \quad u \in C^{1}(D, \mathbf{W}) .
$$

These results can be applied immediately in the various cases discussed so far if we take in the preceding sections $\omega=1$. For an extent force

$$
f_{e} \in T_{e} \operatorname{Emb}\left(\mathcal{B}, \Re^{3}\right)^{*}=C^{1}\left(\mathcal{B}, \Re^{3}\right)^{*}
$$

we may replace in the proposition above $f, u$ and $D$ by $f_{e}, \dot{e}$ and $\mathcal{B}$, respectively. Similarly, we will denote the stresses and force fields corresponding to $\sigma, \Sigma, b$ and $t$ by $\sigma_{e}, \Sigma_{e}, b_{e}$ and $t_{e}$, respectively. In the same fashion, a content force $f_{c} \in C^{1}(\mathcal{B}, \mathbf{V})$ may be represented by content stresses $\sigma_{c}$ and $\Sigma_{c}$, and a force $f_{a}$ may be represented by the stresses $\sigma_{a}$ and $\Sigma_{a}$.

A difficulty arises when one considers the simple body force $f_{m}$ because of the relation $\mathbf{v}=\dot{e} \circ c^{-1}-\mathrm{D} \kappa\left(\dot{c} \circ c^{-1}\right)$. As mentioned in Section 4, we restrict ourselves to forces at smooth configurations of the growing body because of this loss of differentiability.

The relations between the various representatives of a force $f_{\mathcal{B}}$ considered in the previous section, lead to relations between the corresponding stress representatives.

PROPOSITION 6. If any growing subbody is given a force and the collection of forces for the various subbodies is represented by the stress measures $\sigma_{c}, \Sigma_{c}, \sigma_{e}$, $\Sigma_{e}, \sigma_{m}, \Sigma_{m}, \sigma_{a}$ and $\Sigma_{a}$, then

$$
\begin{aligned}
\sigma_{c} & =\sigma_{a}-\sigma_{m}^{*} \circ(\mathrm{D} \kappa \circ c)-\Sigma_{m}^{*} \circ\left(\mathrm{D}^{2} \kappa \circ c\right) \\
\Sigma_{c} & =\Sigma_{a}-\Sigma_{m}^{*} \circ(\mathrm{D} \kappa \circ c) \circ\left(\mathrm{D} c^{-1} \circ c\right)^{*} .
\end{aligned}
$$

Here, the measures $\sigma_{m}^{*}$ and $\Sigma_{m}^{*}$ on $\mathcal{B}$ are the images under $c^{-1}$ of the measures $\sigma_{m}$ and $\Sigma_{m}$ on $c\{\mathcal{B}\}$, respectively.

Proof. Writing the relation $f_{c}(\dot{c})=f_{a}(\dot{c})-f_{m}\left(\mathrm{D} \kappa\left(\dot{c} \circ c^{-1}\right)\right)$ in terms of the corresponding stress measures, we obtain

$$
\begin{aligned}
\int_{\mathcal{B}} \dot{c} d \sigma_{c}+\int_{\mathcal{B}} \mathrm{D} \dot{c} d \Sigma_{c}=\int_{\mathcal{B}} & \dot{c} d \sigma_{a}+\int_{\mathcal{B}} \mathrm{D} \dot{c} d \Sigma_{a} \\
& -\int_{c\{\mathcal{B}\}} \mathrm{D} \kappa\left(\dot{c} \circ c^{-1}\right) d \sigma_{m} \\
& -\int_{c\{\mathcal{B}\}} \mathrm{D}\left(\mathrm{D} \kappa\left(\dot{c} \circ c^{-1}\right)\right) d \Sigma_{m} .
\end{aligned}
$$


One has

$$
\begin{aligned}
\int_{c\{\mathcal{B}\}} \mathrm{D} \kappa\left(\dot{c} \circ c^{-1}\right) d \sigma_{m} & =\int_{\mathcal{B}}(\mathrm{D} \kappa \circ c)(\dot{c}) d \sigma_{m}^{*}, \\
\int_{c\{\mathcal{B}\}} \mathrm{D}\left(\mathrm{D} \kappa\left(\dot{c} \circ c^{-1}\right)\right) d \Sigma_{m} & =\int_{\mathcal{B}}\left(\mathrm{D}\left(\mathrm{D} \kappa\left(\dot{c} \circ c^{-1}\right)\right) \circ c\right) d \Sigma_{m}^{*} .
\end{aligned}
$$

Using

$$
\mathrm{D}\left(\mathrm{D} \kappa\left(\dot{c} \circ c^{-1}\right)\right)=\mathrm{D}^{2} \kappa\left(\dot{c} \circ c^{-1}\right)+\mathrm{D} \kappa\left(\left(\mathrm{D} \dot{c} \circ c^{-1}\right)\left(\mathrm{D} c^{-1}\right)\right),
$$

the relation between the two representations of the force assumes the form

$$
\begin{aligned}
\int_{\mathcal{B}} \dot{c} d \sigma_{c}+\int_{\mathcal{B}} \mathrm{D} \dot{c} d \Sigma_{c}= & \int_{\mathcal{B}} \dot{c} d \sigma_{a}+\int_{\mathcal{B}} \mathrm{D} \dot{c} d \Sigma_{a} \\
& -\int_{\mathcal{B}}(\mathrm{D} \kappa \circ c)(\dot{c}) d \sigma_{m}^{*} \\
& -\int_{\mathcal{B}}\left(\left(\mathrm{D}^{2} \kappa \circ c\right)(\dot{c})\right. \\
& \left.+(\mathrm{D} \kappa \circ c)\left(\mathrm{D} \dot{c}\left(\mathrm{D} c^{-1} \circ c\right)\right)\right) d \Sigma_{m}^{*} .
\end{aligned}
$$

Using the notation $\left(\mathrm{D} c^{-1} \circ c\right)^{*}(\mathrm{D} \dot{c})=\mathrm{D} \dot{c}\left(\mathrm{D} c^{-1} \circ c\right)$ the last equation may be written as

$$
\begin{aligned}
& \int_{\mathcal{B}} \dot{c} d \sigma_{c}+\int_{\mathcal{B}} \mathrm{D} \dot{c} d \Sigma_{c}=\int_{\mathcal{B}} \dot{c}\left(d \sigma_{a}-d \sigma_{m}^{*} \circ(\mathrm{D} \kappa \circ c)-d \Sigma_{m}^{*} \circ\left(\mathrm{D}^{2} \kappa \circ c\right)\right) \\
&+\int_{\mathcal{B}} \mathrm{D} \dot{c}\left(d \Sigma_{a}-d \Sigma_{m}^{*} \circ(\mathrm{D} \kappa \circ c) \circ\left(\mathrm{D} c^{-1} \circ c\right)^{*}\right) .
\end{aligned}
$$

Since the above equality holds not only for the growing body $\mathcal{B}$ but also for the case where the integration is performed over any growing subbody $\mathcal{P}$ of $\mathcal{B}$ it follows (Segev and de Botton, 1991) that

$$
\begin{aligned}
\sigma_{c} & =\sigma_{a}-\sigma_{m}^{*} \circ(\mathrm{D} \kappa \circ c)-\Sigma_{m}^{*} \circ\left(\mathrm{D}^{2} \kappa \circ c\right) \\
\Sigma_{c} & =\Sigma_{a}-\Sigma_{m}^{*} \circ(\mathrm{D} \kappa \circ c) \circ\left(\mathrm{D} c^{-1} \circ c\right)^{*} .
\end{aligned}
$$

We now make the additional assumption that the various stress measures are given in terms of differentiable densities with respect to the volume measure $V_{\mathcal{B}}$ on $\mathcal{B}$. We note that typical treatments of continuum mechanics consider only stresses that are given in terms of differentiable densities with respect to the volume 
measures on the body. We will denote these stress (density) fields by replacing the greek characters with the corresponding roman characters. Thus, we have

$$
\begin{aligned}
d \sigma_{c} & =s_{c} d V_{\mathcal{B}}, \\
d \Sigma_{c} & =S_{c} d V_{\mathcal{B}}, \\
d \sigma_{a} & =s_{a} d V_{\mathcal{B}}, \\
d \Sigma_{a} & =S_{a} d V_{\mathcal{B}}, \\
d \sigma_{m}^{*} & =s_{m}^{*} d V_{\mathcal{B}}, \\
d \Sigma_{m}^{*} & =S_{m}^{*} d V_{\mathcal{B}}, \\
d \sigma_{e} & =s_{e} d V_{\mathcal{B}}, \\
d \Sigma_{e} & =S_{e} d V_{\mathcal{B}} .
\end{aligned}
$$

Using the stress fields we may represent the generalized force as

$$
f_{\mathcal{B}}(\dot{\kappa})=\int_{\partial \mathcal{B}}\left(\dot{c} \cdot t_{c}+\dot{e} \cdot t_{e}\right) d A_{\mathcal{B}}+\int_{\mathcal{B}}\left(\dot{c} \cdot b_{c}+\dot{e} \cdot b_{e}\right) d V_{\mathcal{B}}
$$

where $A_{\mathcal{B}}$ is the area measure on $\partial \mathcal{B}$, the surface force fields are given by

$$
t_{c}=S_{c}(n), \quad t_{e}=S_{e}(n),
$$

and the body force fields are given by

$$
b_{c}=s_{c}-\operatorname{Div} S_{c}, \quad b_{e}=s_{e}-\operatorname{Div} S_{e} .
$$

Similar representations hold for the components $\left(f_{a}, f_{m}\right)$ of the force.

The self forces corresponding to the material force will vanish and the corresponding Cauchy stresses will be be symmetric if one assumes that forces do not perform power in case of a rigid body motion of the body. (See Segev 1984 for the case where the stresses are measures rather than differentiable fields.) The last requirement is usually motivated by the symmetry of the physical space. For the forces associated with the growth of the body such a requirement, leading to the corresponding "balance laws" can be motivated as follows. If we interpret contents of the growing body as unloaded (or other preferred) configurations of the growing body in space, the force $f_{c}$ corresponding to the content should have the same symmetry properties. In other words, no power is associated with infinitesimal change of the unloaded configuration described by rigid body motion. Hence, $\sigma_{c}=0$ and the Cauchy stresses associated with $\Sigma_{c}$ and $S_{c}$ are symmetric.

\section{Acknowledgements}

This work was partially supported by the Paul Ivanier Center for Robotics Research and Production Management, Ben-Gurion University of the Negev. 


\section{References}

Dunford, N. and Schwartz, J.T.:Linear Operators 1, Wiley-Interscience, Chichester, 1971, 43.

Eshelby, J.D.: 'Energy relations and the energy-momentum tensor in continuum mechanics,' in: M.F. Kanninen, W.F. Adler, A.R. Rosenfield, R.I. Jaffee (Editors), Inelastic Behavior of Solids, McGraw-Hill, New York, 1969, pp. 77-115.

Gurtin, M.E.: 'The nature of configurational forces', Archive for Rational Mechanics and Analysis, 1995, (to appear).

Michor, P.W.: Manifolds of Differentiable Mappings, Shiva, London, 1980.

Segev, R.: 'On the definition of forces in continuum mechanics,' in: A. Blaquière, G. Leitmann (Editors), Dynamical Systems and Microphysics Control Theory and Mechanics, Academic Press, 1984, pp. 341-357.

Segev, R.: 'Forces and the existence of stresses in invariant continuum mechanics,' Journal of Mathematical Physics 27 (1986) 163-170.

Segev, R. and de Botton, G.: 'On the consistency conditions for force systems,' International Journal of Nonlinear Mechanics 26 (1991) 47-59. 